\title{
A comparative study among PID structures applied on a Buck converter control
}

\author{
Luís Fabiano Barone Martins ${ }^{1}$, Ricardo Breganon ${ }^{2}$, Uiliam Nelson Lendzion Tomaz Alves ${ }^{3}$, João \\ Paulo Lima Silva de Almeida ${ }^{4}$, and Elenilson de Vargas Fortes ${ }^{5}$
}

\begin{abstract}
In this work, a state-space model and control of a DC-DC Buck converter, considering a continuous operating, are presented. A PID controller is considered in the strategy, that considers a low pass filter in the derivative term. The proposed model is validated by comparing it with a switched model. The PID gains are obtained by the Ziegler-Nichols method. In order to improve the system's performance considering an environment containing high-frequency noises, the modified PID controller is implemented with several configurations.
\end{abstract}

Index Terms-Choppers, Step Down Converter, PID Controller, Ziegler-Nichols Method, Average Model.

\section{INTRODUCTION}

$\mathrm{P}$ OWER electronics is an applied science that aims the static converters study in order to control and convert electrical energy into a compatible signal (voltage level and/or frequency) to determined devices. These converters operate by switching semiconductor devices and they can classified as: AC-DC, AC-AC, DC-AC and DC-DC [1], [2].

DC-DC converters, also known as Choppers, are power electronic circuits with linear and non-linear characteristics, that are composed by resistors, capacitors and inductors and semiconductor switches, respectively [3]. Their applications encompass from power sources for electronic devices to photovoltaic generation plants. By controlling the input voltage switching, DC-DC converters can adjust the magnitude of the output voltage analogously to a AC transformer, adding the possibility of adjusting the output voltage [4].

The mentioned converters operate by the Pulse Width Modulation (PWM) principle, in which a semiconductor switch, actuated by a pulse generator, is used to control the amperage level from the input to the output of the electrical system [2], [4]. This technique aims to reach the highest efficiency as possible (100\%), however, due to the real characteristics (non-ideal) of these semiconductor devices, a typical efficiency related to the converters ranges from $70 \%$ to $95 \%[5]$.
The four classical topologies for non-isolated DC-DC converters are: Cuk, Buck (Step-Down); Boost (Step-Up); and e Buck-Boost [6]. Each one of them has exclusive properties that include the steady-state voltage gain, the source of the input and output currents and the oscillatory characteristics of the output voltage. The most common topology, and probably the simplest, is the Buck (step-down), possibly because its output voltage is always less than the input voltage, with the same polarity and not isolated.

The first step to project an efficient controller to the Choppers is to obtain a complete system's model, including the most part of the non-linearities. A linear model with small signals, by means of a state-space average model around an adequate operation point, is a valid option [7].

In general, the most control techniques applied on Choppers represents a challenging field due to their non-linear and timevarying characteristics [8]. These techniques perform the switching control of the semiconductor devices aiming the maximization of the efficiency related to the power transfer and the good tracking of the output voltage. Several control techniques are used in these converters, although there is a preference for controllers with simple and low cost architecture in industrial applications [9], [10]. In this way, Proportional-Integrative-Derivative (PID) controllers are highlighted [11], [12].

One of the most popular method to adjust the PID gains is the proposed by Ziegler e Nichols (ZN) [13], due to its simple rules and satisfactory performances when it is applied on first order systems [14]. In general, ZN method is used to tune PID controllers under ideal conditions without significant noises and disturbances, which often does not represent a real situation. Therefore, it is necessary to consider their effects in the control algorithm [15].

On the other hand, a filter system is necessary when there are significant noise levels in the control plant. Traditionally, filters are applied on derivative term, due to its characteristic in amplification high-frequency noises.

This work approaches the high-frequency noise effects in DC-DC Buck converter coupled to a PID controller, with a low-pass filter in the derivative term and tuned by $\mathrm{ZN}$ method.

To do this, a linear model to small disturbances of the Buck converter is considered, by means of the average state-space OPEN $0^{\text {ACCESS }}$ 
model and also considering the dynamic of the PID controller. Finally, the system's output response for two different PID gains with the low-pass filter in the derivative term is compared with a standard PID architecture.

The rest of the paper is divided as follows: the PID control structures considered in this work are presented in Section II; in Section III, the mathematical description of the PID controller considering the average model of the Buck converter is addressed; the $\mathrm{ZN}$ method is discussed in Section IV; the main results and conclusions are presented in the Sections V and VI, respectively.

\section{PID CONTROLLER}

The feedback loop in control systems aims to provide the real output signal (controlled variable added to noises, disturbances, and others) of the plant to the control system's input and then to generate a signal to be applied on the manipulated variable, according to the considered control law and aiming to reach a desired reference. The PID controller uses this feedback concept with the proportional $(\mathrm{P})$, integrative (I) and derivative (D) gains, all of them related to the error (difference between the desired reference and the real output signal), which present specific contributions in the control signal calculation [16]. The well-known PID topology, the ISA algorithm [17], is presented in Eq. (1).

$$
u(t)=k_{p}\left(e(t)+\frac{1}{T_{i}} \int_{0}^{t} e(\tau) d \tau+T_{d} \frac{d e(t)}{d t}\right)
$$

where $u$ is the control variable; $e=r-y$ is the error calculation; $r$ is the reference value (set point); $y$ is the controlled variable (system's output); while $T_{i}$ and $T_{d}$ are constants integral and derivative times, respectively, and $k_{p}$ is the proportional gain of the PID controller.

Although simple, the control output computed by the Eq. (1) presents severe implications in real applications, mainly due to the derivative part (related to $T_{d}$ constant time), that carries out noise amplification and then the control variable ( $u$ ) may be unfeasible.

Let $n$ a sinusoidal noise with amplitude and frequency $\omega$ ) given by (2), and $u_{n}$ is its contribution to the derivative term in the control signal, given by (3).

$$
\begin{gathered}
n=a \sin (\omega t) \\
u_{n}=k_{p} T_{d} \frac{d}{d t}[a \sin (\omega t)]=a k_{p} T_{d} \omega \cos (\omega t) \\
=k_{h f} a \cos (\omega t)
\end{gathered}
$$

According to Eq. (3), when $\omega \rightarrow \infty, k_{\mathrm{hf}} \rightarrow \infty$.

In order to avoid unfeasible values (very high values) from derivative part in high frequencies, a common procedure is to limit its bandwidth using a low-pass filter with the transfer function (TF) defined in (4) [18].

$$
G_{f}=\frac{1}{\alpha T_{d} s+1}
$$

According to [16], acceptable values for $\alpha$ ranges from 0.05 to 0.125 . Converting ISA algorithm presented in Eq. (1) into frequency domain and applying the TF of Eq. (4) to derivative term, Eq. (5) can be defined.

$$
U(s)=k_{p}\left[1+\frac{1}{s T_{i}}+\frac{s T_{d}}{s\left(\alpha T_{d}\right)+1}\right] E(s)
$$

In addition to limiting gains of the high frequency components from the error, by the relation $k_{p} / \alpha$, the lowpass filter solves the problem of the non-causality of the ideal PID controller (Eq. (1)) adding a pole in its transfer function.

\section{ZIEGLER-NICHOLS METHOD}

Ziegler and Nichols publish in 1942 a work [19] in which two they describe two strategies to tune P, PI and PID gains. These strategies contemplate a step and frequency response methods. In this work, the step response method is adopted.

Ziegler and Nichols defined as acceptable that the ratio between the amplitude peaks (due to a disturbance in the operation point) in the closed-loop response ( $A_{1}$ and $A_{2}$ in Fig. 1 (a)) is about 4 . However, there is no guarantee that this ratio corresponds to a real system, after tunning process.

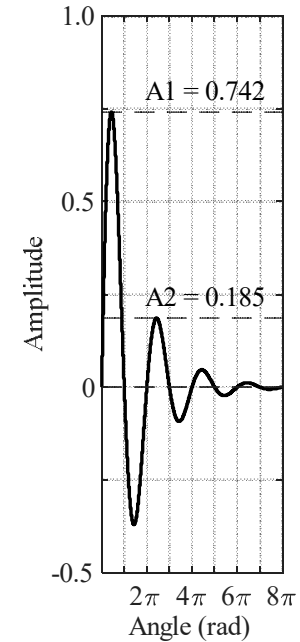

(a)

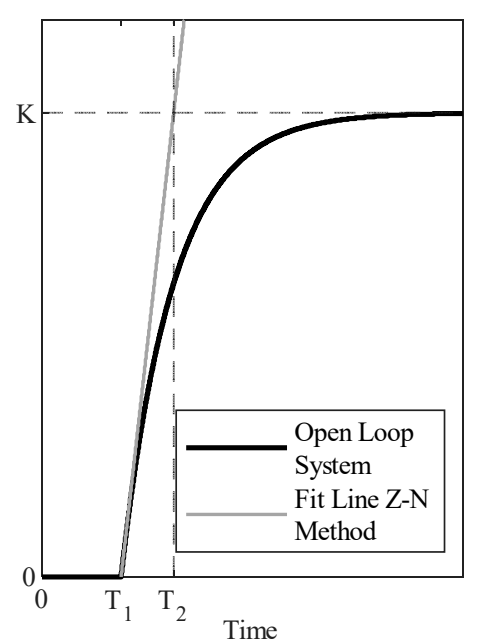

(b)
Fig. 1. (a) Impulse response of a system with transport delay; (b) Step response method.

An illustrative example of the step response method is shown in Fig. 1 (b), considering an open-loop system. This response represents a first-order system with delay transport and its transfer function $H(s)$ is presented in Eq. (6). 


$$
H(s)=\frac{K}{\left(T_{2}-T_{1}\right) s+1} e^{-s T_{1}}
$$

The first-order response (Fig 1 (b)) is composed by two parameters: the time delay $\left(T_{1}\right)$ and the time constant $T_{2}-T_{1}$. They can be determined with a tangent line to the inflection point and by observing its intersections between the axis related to the time and the state-steady value, $K$.

In real time control systems, a wide variety of process plant can be modeled as (6) and from $K, T_{1}$ and $T_{2}$ it is possible to obtain the controller gains as described in TABLE I.

TABLE I

CONTROLLER'S GAINS OBTAINED FROM THE STEP RESPONSE METHOD.

\begin{tabular}{c|c|c|c}
\hline Tipo do controlador & $\mathbf{k}_{\mathbf{p}}$ & $\mathbf{T}_{\mathbf{i}}$ & $\mathbf{T}_{\mathbf{d}}$ \\
\hline $\mathrm{P}$ & $\frac{T_{2}-T_{1}}{T_{1}}$ & & \\
\hline PI & $\frac{9}{10} \frac{T_{2}-T_{1}}{T_{1}}$ & $\frac{10}{3} T_{1}$ & \\
\hline PID & $\frac{6}{5} \frac{T_{2}-T_{1}}{K T_{1}}$ & $2 T_{1}$ & $\frac{1}{2} T_{1}$ \\
\hline \hline
\end{tabular}

IV. PID CONTROLler ADDED TO THE AVERAGE STATE SPACE MODEL

In DC-DC Buck converter, shown in Fig. 2, the input voltage is represented by an ideal DC source $V_{\text {in }}$, while the switch $S$ is a MOSFET transistor. $i_{L}$ is the current through inductor $L, v_{C}$ is the capacitor's voltage $(C)$ and $d \in[0,1]$ is the PWM's duty cycle and it is the control signal of the switch $S . R$ and $D$ are resistor and diode, respectively.

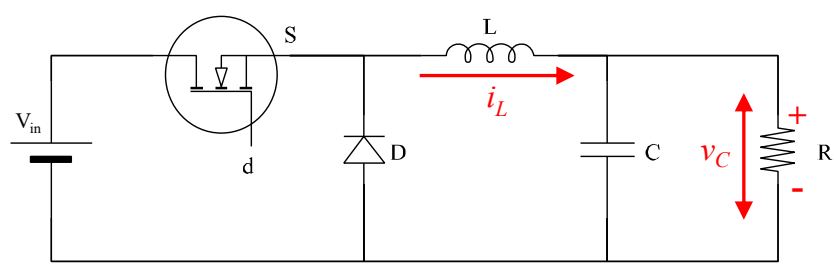

Fig. 2. DC-DC Buck converter.

In order to determine the differential equations of the Buck converter, with switching period $T$, the Kirchhoff's Laws are applied to each functioning state, that is, when $S$ is closed at $[0, d T]$ and when $S$ is open at $[d T, T]$. In this way, the average state space model for the Buck converter [7] is given by Eq. (7)-(8).

$$
\dot{i}_{L}=-\frac{1}{L} v_{C}+\frac{V_{\text {in }}}{L} d
$$

$$
\dot{v}_{C}=\frac{1}{C} i_{L}-\frac{1}{R C} v_{C}
$$

The transfer function in (9) is related to the capacitor voltage under a small duty cycle variation around the operation point of the Buck converter.

$$
G(s)=\frac{\Delta v_{C}(s)}{\Delta d(s)}=\frac{\frac{1}{L C}}{s^{2}+\frac{1}{R C} s+\frac{1}{L C}} V_{\text {in }}
$$

From the Buck converter definition (Eq. (7)-(8)), an expanded state-space model is proposed, including the modified PID dynamics (Eq. (5)).

The Buck converter dynamic with a PID controller can be described by (10).

$$
\begin{aligned}
& \dot{x}=f(x, w, u) \\
& 0=h(x, w, u) \\
& y=g(x, w, u)
\end{aligned}
$$

where $f, h$ and $g$ represent differential, algebraic and output equations, respectively, while $x, w$ and $u$ are the following vectors, respectively: state variables, algebraic vector and system's input.

The use of linear techniques for stability analysis under small disturbances in a dynamic system is a valid approach due to its linearization around an equilibrium point. In this way, assuming small variations around the operation point $\left(x_{0}, w_{0}, u_{0}\right)$, the linearized form of (10) can be described in matrix form as (11).

$$
\left[\begin{array}{c}
\Delta \dot{\mathbf{x}} \\
\mathbf{0} \\
\Delta \mathbf{y}
\end{array}\right]=\left[\begin{array}{lll}
\mathbf{J}_{1} & \mathbf{J}_{2} & \mathbf{B}_{1} \\
\mathbf{J}_{3} & \mathbf{J}_{4} & \mathbf{B}_{2} \\
\mathbf{J}_{5} & \mathbf{J}_{6} & \mathbf{B}_{3}
\end{array}\right]\left[\begin{array}{c}
\Delta \mathbf{x} \\
\Delta \mathbf{w} \\
\Delta \mathbf{u}
\end{array}\right]
$$

In (11), $\mathbf{J}$ matrices are the system's Jacobians at the equilibrium point $\left(x_{0}, w_{0}, u_{0}\right)$. Assuming that $\mathbf{J}_{4}$ is nonsingular, $\Delta \mathbf{w}$ (vector of linearized algebraic variables) can be excluded and then the linear and time-invariant in (11) can be rewritten as (12).

$$
\left[\begin{array}{c}
\Delta \dot{x}_{e} \\
\Delta y
\end{array}\right]=\left[\begin{array}{ll}
A_{e} & B_{e} \\
C_{e} & D_{e}
\end{array}\right]\left[\begin{array}{c}
\Delta x_{e} \\
\Delta u
\end{array}\right]
$$

In (12), the state $\mathbf{A}_{\mathrm{e}}$, input $\mathbf{B}_{\mathrm{e}}$, output $\mathbf{C}_{\mathrm{e}}$ and feedback $\mathbf{D}_{\mathrm{e}}$ matrices can be defined, respectively by Eq. (13)-(16).

$$
\mathbf{A}_{\mathrm{e}}=\mathbf{J}_{1}-\mathbf{J}_{2} \mathbf{J}_{4}^{-1} \mathbf{J}_{3}
$$




$$
\begin{aligned}
& \mathbf{B}_{\mathrm{e}}=\mathbf{B}_{1}-\mathbf{J}_{2} \mathbf{J}_{4}{ }^{-1} \mathbf{B}_{2} \\
& \mathbf{C}_{\mathrm{e}}=\mathbf{J}_{5}-\mathbf{J}_{6} \mathbf{J}_{4}{ }^{-1} \mathbf{J}_{3} \\
& \mathbf{D}_{\mathrm{e}}=\mathbf{B}_{3}-\mathbf{J}_{6} \mathbf{J}_{4}{ }^{-1} \mathbf{B}_{2}
\end{aligned}
$$

For the system addressed in this paper, the output variables are not related to the system's input which implies $\mathbf{D}_{\mathbf{e}}=\mathbf{0}$.

In order to represent the Buck converter with a control about the capacitor voltage $v_{C}$, as in (12), it is necessary to consider each term of the PID dynamic equation (Eq. (5)), individually. From that, Fig. 3 illustrates the considered control structure through block diagram with the algebraic and states variables from (7) and (8).

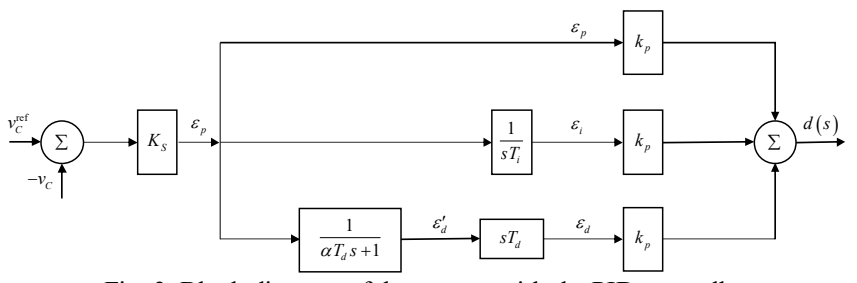

Fig. 3. Block diagram of the system with the PID controller.

From Fig. 3, it is possible to obtain the new state variables, $\varepsilon_{i}$ and $\varepsilon_{d}^{\prime}$, in addiction to the equations that define the algebraic variables $\varepsilon_{p}, \varepsilon_{d}$ and $d(s)$, as shown in Eq. (17)(21), respectively.

$$
\begin{gathered}
\dot{\varepsilon}_{i}=\frac{1}{T_{i}} \varepsilon_{p} \\
\dot{\varepsilon}_{d}^{\prime}=\frac{1}{\alpha T_{d}}\left(\varepsilon_{p}-\varepsilon_{d}^{\prime}\right) \\
\varepsilon_{p}=K_{S}\left(-v_{C}+v_{C}^{\mathrm{ref}}\right) \\
\varepsilon_{d}=\frac{1}{\alpha}\left(\varepsilon_{p}-\varepsilon_{d}^{\prime}\right) \\
d=k_{p}\left(\varepsilon_{p}+\varepsilon_{i}+\varepsilon_{d}\right)
\end{gathered}
$$

Applying the linearization by first order Taylor approximation to Eq. (7)-(8) and (17)-(21) and considering the linearized system's output as $\Delta y=\Delta v_{C}$, it is possible to obtain the matrix formulation for the complete system PID-Buck, as shown in (22), in which the vector of the expanded state variables $\Delta \mathbf{x}_{\mathbf{e}}=\left[\begin{array}{lllll}\Delta i_{L} & \Delta v_{C} & \Delta \varepsilon_{i} & \Delta \varepsilon_{d}^{\prime}\end{array}\right]^{t}$.

$$
\left[\begin{array}{c}
\Delta \dot{i}_{L} \\
\Delta \dot{v}_{C} \\
\Delta \dot{\varepsilon}_{i} \\
\Delta \dot{\varepsilon}_{d}^{\prime} \\
-0 \\
0 \\
0 \\
-\Delta y
\end{array}\right]=\left[\begin{array}{cccc:ccc:c}
0 & -\frac{1}{L} & 0 & 0 & 0 & 0 & \frac{V_{\text {in }}}{L} & 0 \\
\frac{1}{C} & -\frac{1}{R C} & 0 & 0 & 0 & 0 & 0 & 0 \\
0 & 0 & 0 & 0 & \frac{1}{T_{i}} & 0 & 0 & 0 \\
0 & 0 & 0 & -\frac{1}{\alpha T_{d}} & \frac{1}{\alpha T_{d}} & 0 & 0 & 0 \\
\hdashline 0 & -K_{S} & 0 & 0 & -1 & 0 & 0 & K_{S} \\
0 & 0 & 0 & -\frac{1}{\alpha} & \frac{1}{\alpha} & -1 & 0 & 0 \\
0 & 0 & k_{p} & 0 & k_{p} & k_{p} & -1 & 0 \\
0 & 1 & 0 & 0 & 0 & 0 & 0 & 0
\end{array}\right]\left[\begin{array}{c}
\Delta i_{L} \\
\Delta v_{C} \\
\Delta \varepsilon_{i} \\
\Delta \varepsilon_{d}^{\prime} \\
-\frac{1}{\varepsilon_{p}} \\
\Delta \varepsilon_{d} \\
\Delta d \\
\Delta v_{C}^{- \text {ref }}
\end{array}\right]
$$

The submatrices $\mathbf{J}$ and $\mathbf{B}$ are used to determine $\mathbf{A}_{\mathbf{e}}, \mathbf{B}_{\mathbf{e}}$ and $\mathbf{C}_{\mathbf{e}}$, from (22) and (13)-(16), resulting (23)-(25).

$$
\begin{gathered}
\mathbf{A}_{\mathbf{e}}=\left[\begin{array}{cccc}
0 & -\frac{1}{L}-\frac{V_{i} k_{p}(\alpha+1) K_{S}}{\alpha L} & \frac{V_{i} k_{p}}{L} & -\frac{V_{i} k_{p}}{\alpha L} \\
\frac{1}{C} & -\frac{1}{R C} & 0 & 0 \\
0 & -\frac{K_{S}}{T_{i}} & 0 & 0 \\
0 & -\frac{K_{S}}{\alpha T_{d}} & 0 & -\frac{1}{\alpha T_{d}}
\end{array}\right] \\
\mathbf{B}_{\mathbf{e}}=\left[\begin{array}{cccc}
\frac{V_{i} k_{p}(\alpha+1) K_{S}}{\alpha L} & 0 & \frac{K_{S}}{T_{i}} & \frac{K_{S}}{\alpha T_{d}}
\end{array}\right]^{t} \\
\mathbf{C}_{\mathbf{e}}=\left[\begin{array}{llll}
0 & 1 & 0 & 0
\end{array}\right]
\end{gathered}
$$

From Eqs. (23)-(25), it is possible to represent the dynamic model of the Buck converter in matrix form and compact, when it is under small disturbances around an operating point $\left(\mathbf{X}_{\mathrm{e}}, v_{C}^{\text {ref }}\right)$.

The transfer function $\left(G_{P}(s)\right)$, aiming the control of voltage $v_{C}$, is obtained according to the model parameters in state space, assuming that the disturbance inputs are null, as in Eq. (26).

$$
G_{P}(s)=\frac{\Delta v_{C}(s)}{\Delta v_{C}^{\text {ref }}(s)}=\mathbf{C}_{\mathbf{e}}\left(s \mathbf{I}-\mathbf{A}_{\mathbf{e}}\right)^{-1} \mathbf{B}_{\mathbf{e}}
$$

In this work, results obtained considering the both PID (standard) and modified, according to Eq. (1) and (5), respectively, are compared, in order to validate the approach addressed in this paper. For that, the transfer function of each compared controller, defined in (27) and (28), with the constants $a_{0}, a_{1}, a_{2}$ and $a_{3}$, is defined in Eq. (29)-(32). 


$$
G_{P 1}(s)=\frac{a_{0}\left(T_{i} T_{d} s^{2}+T_{i} s+1\right)}{s^{3}+a_{2} s^{2}+a_{1} s+a_{0}}
$$

$$
\begin{gathered}
G_{P 2}(s)=\frac{1}{\alpha T_{d}} a_{0}\left[(\alpha+1) T_{i} T_{d} s^{2}+\left(T_{i}+\alpha T_{d}\right) s+1\right] \\
s^{4}+\frac{1}{\alpha T_{d}} a_{3} s^{3}+\frac{1}{\alpha T_{d}}\left(a_{2}+\alpha T_{d} a_{1}\right) s^{2}+\frac{1}{\alpha T_{d}}\left(a_{1}+\alpha T_{d} a_{0}\right) s+\frac{1}{\alpha T_{d}} a_{0} \\
a_{0}=\frac{1}{L C} \frac{k_{p} K_{S} V_{i}}{T_{i}} \\
a_{1}=\frac{1}{L C}\left(k_{p} K_{S} V_{i}+1\right) \\
a_{2}=\frac{1}{L C}\left[k_{p} K_{S} V_{i} T_{d}+\frac{L}{R}\right] \\
a_{3}=\frac{1}{L C}\left(L C+\alpha T_{d} \frac{L}{R}\right)
\end{gathered}
$$

\section{RESUlts}

The proposed system, based on the transfer functions of Buck converter, $G(s)$, and PID controller, $G_{C}(s)$, and considering the voltage sensor gain, $K_{S}$, can be represented

\begin{tabular}{|c|c|}
\hline PARAMETER & VALUE \\
\hline Input voltage $\left(V_{\text {in }}\right)$ & $12 \mathrm{~V}$ \\
\hline Output voltage $\left(v_{C}\right)$ & $5 \mathrm{~V}$ \\
\hline Inductance $(L)$ & $2.4 \mathrm{mH}$ \\
\hline Output capacitance ( $C$ ) & $5.6 \mu \mathrm{F}$ \\
\hline Load resistance $(R)$ & $10 \Omega$ \\
\hline PWM frequency $(f)$ & $10 \mathrm{kHz}$ \\
\hline
\end{tabular}
through the diagram of Fig. 4.

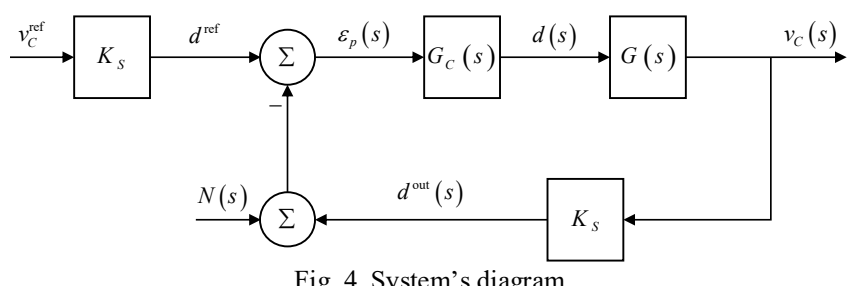

In Fig. 4, $N(s)$ represents the measurement noises of $v_{C}$ and $G_{C}(s)$ encompasses Eq. (27)-(32) and the characteristics of the Buck converter shown in TABLE II.

TABLE II
With the output power of $10 \mathrm{~W}$, the ripples of the output voltage, $\delta v_{C}$, and of the inductor's current, $\delta i_{L}$, are $5.39 \%$ and $6.04 \%$, respectively, which leads the converter to operate in continuous mode.

In order to validate the average state-space model of the Buck converter, represented by Eq. (7) and (8), the simulation software PSIM ${ }^{\circledR}$ was used. To do that, all parameters of TABLE II were considered to obtain the switched model response. Furthermore, a $5 \mathrm{~V}$ step was applied in the reference voltage of the switched models, aiming to obtain the openloop response. The respective responses under disturbances are presented in Fig. 5.

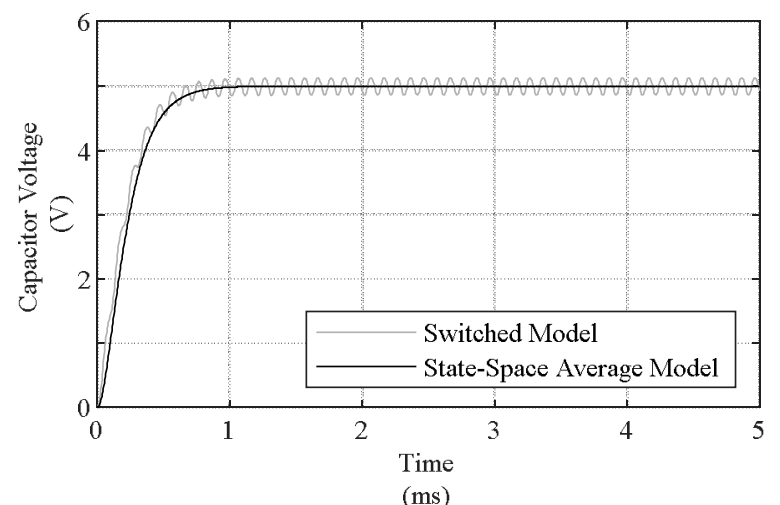

Fig. 5. Transfer functions' response related to the state-space and switched model.

It is important to highlight in Fig. 5 that the state-space average model is a satisfactory representation of the converter's dynamic under low frequency. The suppression of high frequency components of the switched model, due to the discontinuity of the switching process, occurs with the use of average values of the instantaneous variables for a switching period of the power switch $S$ of Fig. 2 .

After validation of the average model, the response of the open-loop transfer function not compensated was obtained (Fig. 6), considering $G_{C}(s)=1$ and $N(s)=0$. In this way, a step of 0.415 was applied in the duty-cycle of PWM reference $\left(d^{\text {ref }}\right)$. That response is presented in Fig. 6 and it can be represented by (6) with a line tangent to its inflection point.

The constants of static gain, transport delay and apparent time (Eq. (6)) can be determined by inspection of Fig. 6, that is, $K=0.415, T_{1}=32 \mu \mathrm{s}$ and $T_{2}-T_{1}=322 \mu \mathrm{s}$. Based on these values and according to the TABLE I, the PID parameters are presented in TABLE III.

TABLE III

GAIN AND TIME CONSTANTS OF PID CONTROLLER BY MEANS OF ZIEGLERNICHOLS METHOD.

\begin{tabular}{c|c|c|c}
\hline \hline Controller & $\boldsymbol{k}_{p}$ & $\boldsymbol{T}_{\boldsymbol{i}}(\boldsymbol{\mu} \mathrm{s})$ & $\boldsymbol{T}_{\boldsymbol{d}}(\boldsymbol{\mu} \mathrm{s})$ \\
\hline PID & 29 & 64 & 16 \\
\hline \hline
\end{tabular}




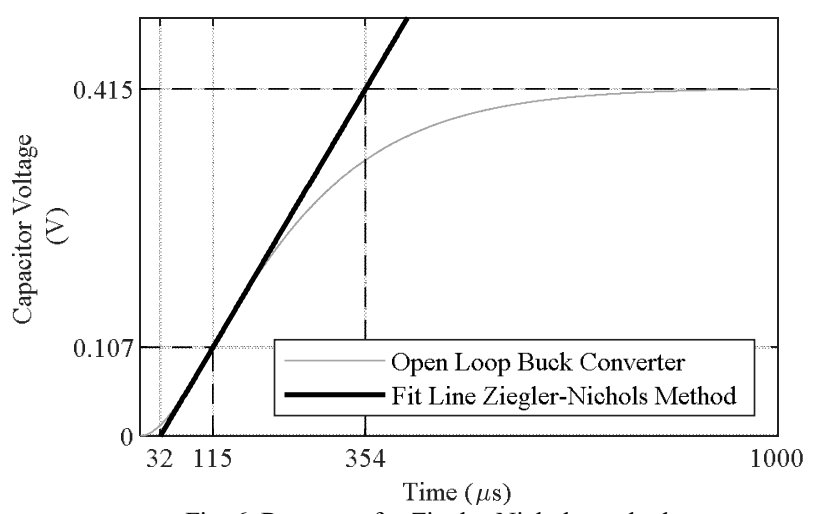

Fig. 6. Response for Ziegler-Nichols method.

Applying the constants of TABLE I and III in the closed-loop transfer function presented in (27), that is, considering a standard PID ( $\alpha=0)$, it is possible to determine the constants about damping $(\xi=-0.391)$ and the damped frequency $\left(\omega_{d}=36.4 \mathrm{krad} / \mathrm{s}\right)$ of the oscillatory mode caused by the pair of conjugated complex poles. The time response with $v_{C}^{\text {ref }}=5$ $\mathrm{V}$ is presented in Fig. 7.

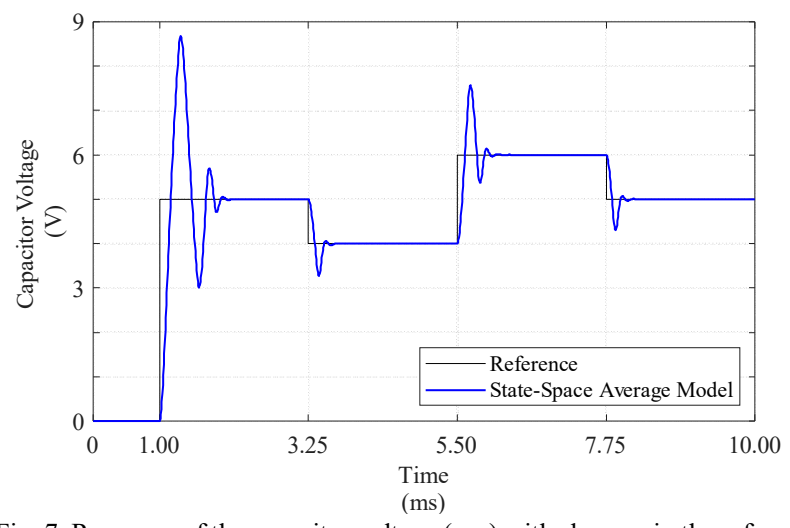

Fig. 7. Response of the capacitor voltage $\left(v_{C}\right)$ with changes in the reference voltage $\left(v_{C}^{\text {ref }}\right)$.

From the response presented in Fig. 7, it is possible to conclude that the controlled variable presents low accommodation time for starting $\left(T_{a}<1 \mathrm{~ms}\right)$ and for disturbances around the equilibrium point $\left(T_{a}<0.7 \mathrm{~ms}\right)$, however the percentual overshoot is higher than $60 \%$. Therefore, if the designer aims a better performance for overshoot characteristic, a fine tuning would be necessary in the PID gains.

According to the literature [16]-[18], a standard PID controller requires a special attention in its derivative term when it is applied in real process. In order to mitigate this situation, a modified structure is considered in this work, in which a low-pass filter is added to derivative term, as presented in (5). To compare the performance between the standard and modified PID architectures, a Gaussian noise (average value equal to zero, components at frequencies up to
2.5 $\mathrm{MHz}$ and standard deviation $\sigma=0.1$ ) is inserted into the system.

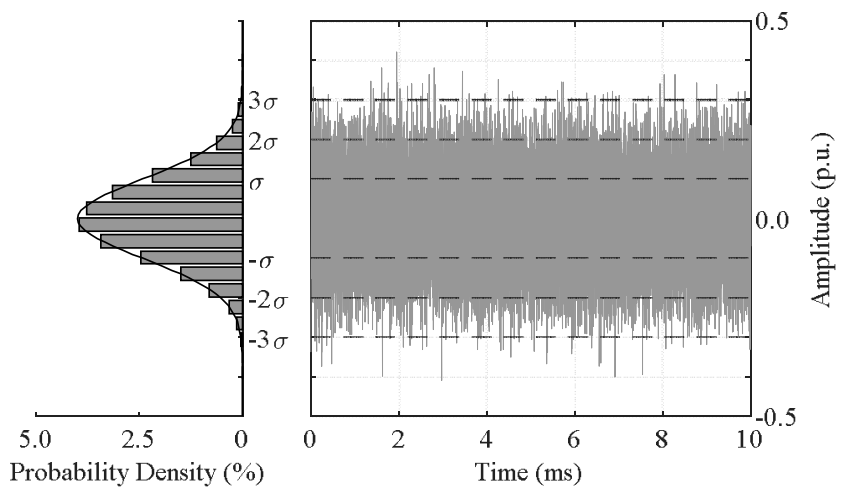

(a)

(b)

Fig. 8. (a) Density and probability function; (b) Gaussian noise.

The response of the density and probability function is shown in Fig. 8 (a), while in Fig. 8 (b) is the Gaussian noise along the time (applied in $N(s)$ ).

To compare the performance between the considered standard and modified PID controllers, 3 levels of $\alpha$ (Eq. (28) ) are considered: $\alpha=0$ (PID standard in (27)); $\alpha=0.05$; and $\alpha=0.125$. The last two values are chosen based on [16]. The system's responses to small disturbances around the operation point, considering the two mentioned controllers are presented in Fig. 9.

In the first experiment ( $\alpha=0)$, the obtained response is the dotted line (Fig. 9), which suggests the difficulty of the control action to follow the reference quickly, when this characteristic is compared to the other cases. In the following experiments, with $\alpha=0.05$ and $\alpha=0.125$, the responses are presented in blue and black colors, respectively. It is reasonable to conclude that the voltage $v_{C}$ is faster when $\alpha=0.125$, in which there is a greater rejection of high-frequency disturbances due to the modified PID. This effect can be explained due to the lower cutoff frequency of the low-pass filter with $\alpha=0.125(79 \mathrm{kHz})$, when it is compared to $\alpha=0.05(198 \mathrm{kHz})$.

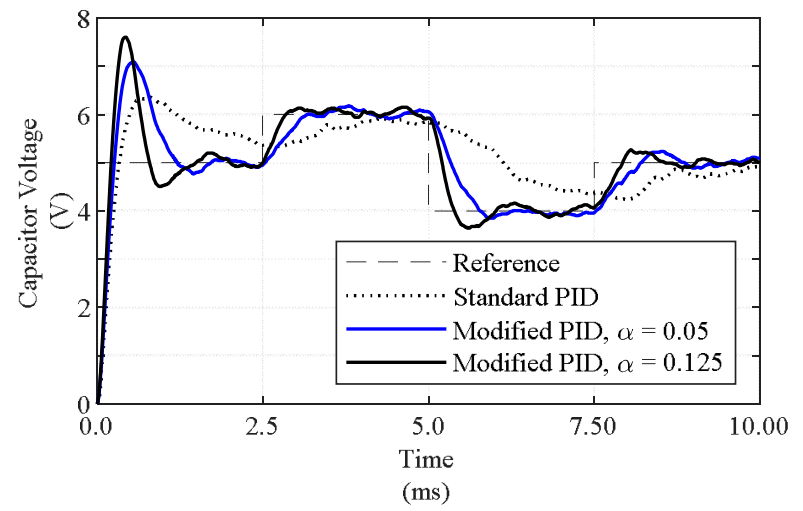

Fig. 9. Response of the capacitor voltage $\left(v_{C}\right)$ to the changes in the reference voltage $\left(v_{C}^{\text {ref }}\right)$. 
It is important to notice that the increase of $\alpha$ causes a higher overshoot in the system's response related to the reference voltage, what is expected due to the decrease in the derivative term.

\section{CONCLUSIONS}

A modified PID controller (a low-pass filter is added to the derivative term) was considered in the state-space average model of a DC-DC Buck converter. From the expanded state equations, that is, adding the controller dynamics, two closedloop transfer functions were obtained, related to the use of standard and the modified PID controllers, respectively.

In order to validate the proposed model, its step response was compared to the switching model available on PSIM ${ }^{\circledR}$ software and the obtained results show equivalent dynamics.

The PID controller was tuned by means of Ziegler-Nichols method. Is can be observed that, from a step input reference, the output voltage reaches acceptable performance, according to the project requirements adopted in this work.

Aiming the minimization of a high frequency noises effects, a low-pass filter was considered in the derivative term of the PID controller. Its performance was compared to a standard PID, according to the literature. From the presented results, it can be concluded that the system's output shows faster response, when it is compared to the other addressed cases.

In future works, we intend to consider other control techniques with optimization methods, aiming to minimize the overshoot and the stabilization time.

\section{REFERENCES}

[1] N. Mohan and M. P. E. R. I\& Education, First Course on Power Electronics and Drives. MNPERE, 2003.

[2] B. K. Bose, "Power electronics-a technology review," Proc. IEEE, 1992, doi: $10.1109 / 5.158603$.

[3] R. B. Ridley, "A New, Continuous-Time Model For Current-Mode Control," IEEE Trans. Power Electron., 1991, doi: $10.1109 / 63.76813$.

[4] N. Mohan, T. M. Undeland, and W. P. Robbins, Power electronics: converters, applications, and design, no. v. 1. John Wiley $\ \&$ Sons, 2003.

[5] R. W. Erickson, "DC-DC Power Converters," in Wiley Encyclopedia of Electrical and Electronics Engineering, American Cancer Society, 2007

[6] E. Van Dijk, J. N. Spruijt, D. M. O'Sullivan, and J. B. Klaassens, "PWM-switch modeling of DC-DC converters," IEEE Trans. Power Electron., vol. 10, no. 6, pp. 659-665, 1995, doi $10.1109 / 63.471285$.

[7] R. D. Middlebrook and S. Cuk, "A general unified approach to modelling switching-converter power stages," in 1976 IEEE Power Electronics Specialists Conference, 1976, pp. 18-34, doi: 10.1109/PESC.1976.7072895.

[8] S. Buso, "Design of a robust voltage controller for a buck-boost converter using $/ \mathrm{spl} \mathrm{mu/-synthesis,"} \mathrm{IEEE} \mathrm{Trans.} \mathrm{Control} \mathrm{Syst.}$ Technol., vol. 7, no. 2, pp. 222-229, 1999, doi: 10.1109/87.748148.

[9] C. K. Tse and K. M. Adams, "Quasi-linear modeling and control of DC-DC converters," IEEE Trans. Power Electron., vol. 7, no. 2, pp. 315-323, 1992, doi: $10.1109 / 63.136248$

[10] A. J. Forsyth and S. V. Mollov, "Modelling and control of DC-DC converters," Power Eng. J., vol. 12, no. 5, pp. 229-236, 1998, doi: 10.1049/pe: 19980507.
[11] S. Seshagiri, E. Block, I. Larrea, and L. Soares, "Optimal PID design for voltage mode control of DC-DC buck converters," in 2016 Indian Control Conference (ICC), Jan. 2016, pp. 99-104, doi: 10.1109/INDIANCC.2016.7441112.

[12] D. E. Rivera, M. Morari, and S. Skogestad, "Internal model control: PID controller design," Ind. Eng. Chem. Process Des. Dev., vol. 25, no. 1, pp. 252-265, Jan. 1986, doi: 10.1021/i200032a041.

[13] J. G. Ziegler and N. B. Nichols, "Optimum Settings for Automatic Controllers," J. Dyn. Syst. Meas. Control, vol. 115, no. 2B, pp. 220 222, 1993, doi: $10.1115 / 1.2899060$.

[14] C. C. Hang, K. J. Astrom, and W. K. Ho, "Refinements of the Ziegler-Nichols tuning formula," IEE Proc. D - Control Theory Appl., vol. 138, no. 2, pp. 111-118, 1991, doi: 10.1049/ipd. 1991.0015

[15] S. Agrawal, V. Kumar, K. P. S. Rana, and P. Mishra, "Optimization of PID controller with first order noise filter," in 2015 International Conference on Futuristic Trends on Computational Analysis and Knowledge Management (ABLAZE), 2015, pp. 226-231, doi: 10.1109/ABLAZE.2015.7154996.

[16] K. J. Äström and T. Hägglund, Advanced PID control. Research Triangle Park, NC: ISA-The Instrumentation, Systems, and Automation Society, 2006.

[17] A. O'Dwyer, Handbook of PI and PID controller tuning rules, 3rd ed. Covent Garden, London: Imperial College Press, 2009.

[18] M. A. Johnson et al., PID control: New identification and design methods. London: Springer-Verlag, 2005.

[19] J. G. Ziegler and N. B. Nichols, "Optimum Settings for Automatic Controllers," The American Society of Mechanical Engineers (ASME), vol. 64, pp. 759-768, 1942.

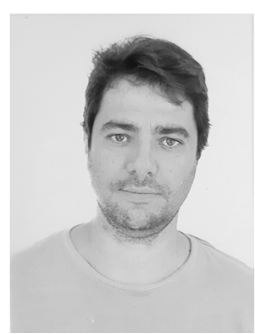

Luís Fabiano Barone Martins is Professor at the Federal Institute of Paraná, Jacarezinho, is graduated in Electrical Engineering from São Paulo State University (UNESP), Campus Ilha Solteira, MSc. degree in Electrical Engineering and $\mathrm{PhD}$. degree in Electrical Engineering from UNESP Campus Bauru and Ilha Solteira, respectively. His research interests include Electrical Power Systems, Power Electronics, Bio-Inspired Optimization Metaheuristics, Industrial Automation and Dynamic Systems Control.

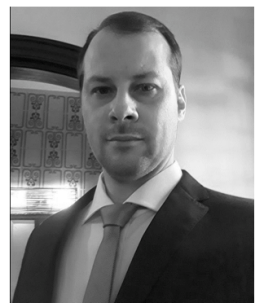

Ricardo Breganon is Professor at the Federal Institute of Paraná, Jacarezinho, holds degree in Production Engineering from Estacio de Sa University and in Mechanical Technology from Federal Technological University of Paraná, MSc. degree in Mechanical Engineering and $\mathrm{PhD}$. degree in Mechanical Engineering with a concentration in Aeronautical from School of Engineering of São Carlos University of São Paulo. His research interests include Industrial Automation and Dynamic Systems Control.

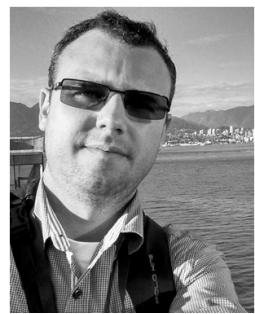

Uiliam Nelson L. T. Alves is graduated in Control and Automation Engineering from UniCesumar, Maringá, PR, Brazil (2011). MSc. and PhD. in Electrical Engineering from UNESP (Universidade Estadual Paulista), Campus of Ilha Solteira, SP, Brazil in 2014 and 2017, 
respectively. At this moment, he is Control and Industrial Processes professor in IFPR (Instituto Federal de Educação, Ciência e Tecnologia do Paraná), campus Jacarezinho. His research interests include Fuzzy modeling and control, Robust and nonlinear control.

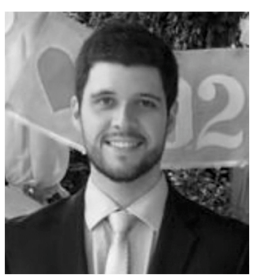

João Paulo Lima Silva de Almeida received the B.Sc. degree in Industrial Automation (2011), the M.Sc. degree in Electrical Engineering (2014) and the Ph.D. degree in Electrical Engineering (2019) from Federal University of Technology - Paraná. He has been with the Federal Institute of Paraná, since 2012. His research interests include intelligent systems to model and control dynamic systems.

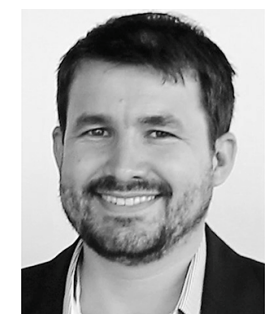

Elenilson de Vargas Fortes received the degree in mathematics in 2004 from the Federal University of Espírito Santo, São Mateus, Brazil, the M.Sc. degree in mathematics in 2007 from the Federal University of Brasília, Brasília, Brazil, and the Ph.D. degree in electrical engineering in 2016 from the São Paulo State University, Ilha Solteira, Brazil. He is currently a professor at the Goiás Federal Institute of Education, Science, and Technology, Jataí, Brazil. His current research interests include small-signal stability analysis in power systems. 